\title{
Gobierno abierto para la modernización del Estado
}

\author{
Open government for the \\ modernization of the State
}

Rafael Enrique Valenzuela¹, J. Ramón Gil-García²

G obierno abierto se refiere a una forma muy particular de gobernar y gestionar las políticas públicas. En un contexto de gobernanza democrática, que se caracteriza por la transparencia, la participación ciudadana y la colaboración entre gobiernos y ciudadanos, el gobierno abierto crea escenarios que se suponía solo eran posibles en sociedades anglosajonas. Los gobiernos de Latinoamérica y el Caribe muestran signos de reconocimiento a nuevas realidades, en las cuales el gobierno ya no decide asuntos colectivos de forma unilateral. Ahora coexiste con otros actores de la sociedad civil y el mercado, otorgando validez a nuevos mecanismos y estrategias para decidir los asuntos públicos de forma conjunta. Este número especial está compuesto de dos artículos y tres reseñas de libros recientes que abordan el gobierno abierto desde perspectivas académicas y de comunidades de practicantes.

Los artículos del número 56 de NOESIS, analizan importantes aspectos de una panorámica global en cuanto a las discusiones teóricas y empíricas del gobierno abierto, a partir de dos esferas, una académica y otra empírica de política pública. El artículo de Diego Barría Traverso, Bastián González-Bustamante y Carla Cisternas Guasch, investigadores de la Universidad de Santiago de Chile, ofrece una visión del gobierno abierto desde la esfera académica. Postulan una metodología innovadora basada en el análisis de redes de citación de textos académicos. Ellos usan un mo-

1- Nacionalidad: Mexicana. Grado: Doctor. Especialización: Política pública. Adscripción: Universidad Autónoma de Ciudad Juárez. Correo electrónico: rafael.valenzuela@uacj.mx. (O) ORCID: http://orcid.org/0000-0002-5898-9590

2- Nacionalidad: Mexicana. Grado: Doctor. Especialización: Administración y políticas públicas. Adscripción: Universidad Estatal de Nueva York en Albany. Correo electrónico: jgil-garcia@ctg.albany.edu. (D) ORCID: http://orcid.org/0000-0003-3236-0281 
delo estadístico para evaluar las determinantes de centralidad de artículos especializados que se han publicado sobre gobierno abierto, específicamente en Internet. Al margen de los hallazgos que comparten los autores, se destaca la riqueza bibliográfica obtenida en la revisión de literatura en español, al dibujar un mapa completo de los enfoques y corrientes teóricas que proponen en la agenda pública el reto de gobernar en tiempos de gobierno abierto.

Alan Hudson y Jorge Florez, Director Ejecutivo y Coordinador de Investigación de Global Integrity, respectivamente, nos presentan la esfera empírica valiéndose de una visión de política pública. Su artículo muestra los avances más recientes hacia una Gobernanza Fiscal Abierta en América Latina, a través del análisis de los compromisos vinculados con el eje gestión eficaz de los recursos públicos, uno de los cinco que postula el modelo de la Alianza Global por el Gobierno Abierto (OGP, por sus siglas en inglés). Los autores muestran, con base en herramientas e iniciativas internacionales, los esfuerzos que a nivel global se realizan para promover mejoras en el uso de los recursos públicos. La contribución de Global Integrity al debate de la apertura gubernamental, se realizó a través de un modelo de cuatro capas construido por los autores: 1) las políticas y regulaciones, 2) los datos, 3) oportunidades para incidir en las decisiones públicas y 4) las capacidades gubernamentales y ciudadanas. Para Alan Hudson y Jorge Florez, el gobierno abierto implica la implementación de los principios de transparencia, participación ciudadana y rendición de cuentas, y su alcance tiene un valor práctico y transformador, en las relaciones entre ciudadanía y gobierno.

Por último, se publican tres reseñas en este número especial, elaboradas por Lilian Salado Rodríguez, académica de la Universidad Estatal de Sonora; Jehú Jonathan Ramírez Camberos, profesor del Instituto Sonorense de Administración Pública, y; Edgar Alejandro Ruvalcaba Gómez, investigador doctorando de la Universidad Autónoma de Madrid. Cada reseña destaca por mostrar los textos más recientes de gobierno abierto hasta 2016, y que se pueden considerar referencias obligadas para los y las especialistas en gobierno abierto alrededor del mundo y particularmente en Iberoamérica. 